\title{
Carnets
}

Revue électronique d'études françaises de l'APEF

Deuxième série - 8 | 2016

Du Français en cause aux causes du français

\section{Les politiques linguistiques européennes}

Un plus pour le Français?

\section{Filomena Capucho}

\section{OpenEdition}

Journals

Édition électronique

URL : http://journals.openedition.org/carnets/1844

DOI : $10.4000 /$ carnets. 1844

ISSN : 1646-7698

Éditeur

APEF

Référence électronique

Filomena Capucho, «Les politiques linguistiques européennes 》, Carnets [En ligne], Deuxième série - 8 | 2016, mis en ligne le 30 novembre 2016, consulté le 10 décembre 2020. URL : http://

journals.openedition.org/carnets/1844; DOI : https://doi.org/10.4000/carnets.1844

Ce document a été généré automatiquement le 10 décembre 2020.

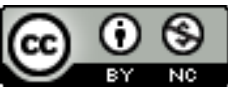

Carnets est mis à disposition selon les termes de la licence Creative Commons - Atribution - Pas d'utilisation commerciale 4.0 International. 


\section{Les politiques linguistiques européennes}

Un plus pour le Français?

Filomena Capucho

\section{Introduction}

1 Réfléchir sur le rôle actuel du français, en contexte portugais, européen ou mondial, nous oblige à le placer dans le cadre des tendances idéologiques, politiques et économiques qui déterminent les politiques linguistiques qui sous-tendent, de nos jours, les représentations sociales sur les langues, leur utilité et leur apprentissage en milieu formel ou informel.

2 Cet article présentera ainsi une analyse de l'évolution des politiques linguistiques européennes mises en œuvre depuis le début $d u \mathrm{xxI}^{\mathrm{e}}$ siècle, tout en réfléchissant sur l'importance du développement du multilinguisme des états et du plurilinguisme des citoyens. Dans ce cadre, j'interrogerai la place que les langues étrangères pourront occuper dans un monde culturellement diversifié, où le dialogue interculturel et le respect des diversités est essentiel pour le maintien de la paix et la construction d'une citoyenneté plurielle, dans un cadre de liberté et de démocratie. Je m'arrêterai ensuite plus longuement sur l'analyse du rôle que les approches plurielles peuvent jouer par rapport à l'intégration du français dans sa famille linguistique - les langues romanes dont l'apprentissage pourra revitaliser l'intérêt social porté à la langue française et lui redonner un nouvel élan.

\section{Mondialisation ou diversité(s)?}

3 Même si certains auteurs, tels que Friedman (2000), estiment que le processus de mondialisation a commencé il y a des siècles, en particulier depuis l'âge des grandes découvertes maritimes, nous pouvons situer les changements majeurs à ce niveau depuis la fin $\mathrm{du} \mathrm{xx}^{\mathrm{e}}$ siècle, provoqués par des événements historiques comme la chute du mur de 
Berlin, mais, surtout, par la grande transformation due au développement des technologies de l'information et de la communication: l'arrivée du système d'exploitation Windows, l'émergence de Netscape (1995) et l'extension du potentiel de l'Internet autour l'an 2000 (Munshi, 2006 : 261-267). Nous assistons ainsi, au cours des 15 dernières années, à une révolution technologique centrée sur les processus d'information, qui «remodèle à un rythme accéléré les fondements matériels de la société » (Castells, $1998: 21)$.

4 Toutefois, si la mondialisation est une réalité indéniable, elle n'implique pas nécessairement la soumission à un monde unique, uniforme, lisse et soumis. En fait, comme Stiglitz (2006: 4) le soutient, le problème n'est pas dans la mondialisation, mais dans la façon dont la mondialisation a été gérée et dont certains souhaitent nous la montrer pour mieux l'imposer. La mondialisation peut, au contraire, garantir le respect des diversités car " [a] tendência da globalização - um mundo uno, interconectado e interdependente - supõe simultaneamente, e como parte de um mesmo processo, a reafirmação da diversidade sócio-económico-cultural e das identidades locais e nacionais » (Bittencourt, $2008: 2$ ). Ainsi, la mondalisation peut aller de pair avec la liberté d'existence de chacun et de ses choix personnels, l'authenticité, permettant le slogan longtemps utilisé par la Commission Européenne : « Unité dans la diversité ». En contexte européen, ce sera donc la reconnaissance d'un immense héritage culturel qui nous permettra, aujourd'hui encore, (re)penser notre identité: «il semble que la véritable identité de l'Europe réside dans son immense richesse culturelle et dans sa capacité à évoquer une dimension imaginale du pouvoir » (Bellini, 2008 : 12).

\section{Un cadre pluriel de communication}

Dans cet espace européen de plus en plus élargi et multiculturel, mais qui cherche à préserver les identités individuelles et collectives, il faut donc faire face à une nouvelle problématique au niveau de la communication entre les états, les entreprises et les individus :

As a result of enlargement, the Single Market and increased mobility within the EU, the revival of the regions, the advent of the knowledge society, migration into the $\mathrm{EU}$, and globalisation, this multilingual challenge has reached a completely new dimension - in terms of size, complexity, and policy relevance (Commission of the European Communities, 2007: 6).

6 Nombreux sont les défis à relever. Ils couvrent non seulement la communication réelle, mais tous les aspects qui découlent du fait que l'« unité dans la diversité » dont on parle existe non seulement dans son ensemble, mais aussi dans chacun des États qui la composent, constituant ce que Vertovec (2006) a appelé « super-diversité » :

Whereas this was formerly primarily seen in terms of interpersonal exchanges between people residing in different Member States, practically all EU Member States have by now become multilingual and multicultural societies themselves, requiring strategies at local / regional / Member State level for facilitating communication across language and cultural boundaries (Commission of the European Communities, 2007: 6).

7 En 2016, l'Union européenne compte plus de 508 millions de personnes, dont plus de 20 millions d'immigrants ${ }^{1}, 28$ États membres ${ }^{2}, 3$ alphabets et 24 langues officielles. En outre, on compte encore dans le patrimoine européen plus de 60 langues parlées dans des régions spécifiques ou par des groupes spécifiques; le phénomène de l'immigration y a 
apporté plus de 100 langues différentes. Une étude réalisée dans le cadre du projet VALEUR $^{3}$ estime également que, en fait, dans les 21 pays qui formaient la base du rapport de travail produit, un total de 600 langues devrait être pris en compte (y compris en plus des langues officielles, les langues régionales ou minoritaires, les langues de l'immigration, les langues non-territoriales et les différentes langues des signes) : « Pour ce qui est des 21 pays participants, un total de l'ordre de 600 langues pourrait donc se révéler plus exact » (McPake \& Tinsley, 2007 : 29).

8 Le nouveau cadre européen se caractérise donc par une diversité croissante dont la gestion est de plus en plus nécessaire. Dans le rapport du groupe d'intellectuels réunis en 2008 à l'initiative de la Commission européenne, Amin Maalouf mentionne clairement ce problème :

[...] pour toute société humaine, la diversité linguistique, culturelle, ethnique ou religieuse présente à la fois des avantages et des inconvénients, une source de richesses mais également une source de tensions; l'attitude sage consiste à reconnaître la complexité du phénomène, en s'efforçant d'en maximiser les effets positifs et d'en minimiser les effets négatifs (Maalouf, $2008: 3$ ).

\section{Options de politique linguistique en Europe : le multilinguisme}

9 Il faut donc clairement suivre des options sociales, culturelles et linguistiques afin de valoriser le potentiel de richesse et de minimiser les conflits qui pourront survenir. À partir de 2000, avec la Déclaration de Lisbonne, renforcée en 2002 avec la Déclaration de Barcelone ${ }^{4}$, l'option politique de l'Union européenne a visé le respect et la défense du multilinguisme. Depuis, toute une série de mesures politiques linguistiques ont été prises, y compris non seulement la production de documents soutenant cette option, comme la mise en œuvre d'une politique linguistique éducative pour le développement du plurilinguisme des citoyens ${ }^{5}$ : "At the Barcelona European Council in 2002, at the suggestion of the European Commission, the Member States set themselves the objective of European citizens learning at least two foreign languages » (Orban, $2007: 7$ ).

Il s'agit d'une option politique que plusieurs arguments justifient :

- Idéologiques - le multilinguisme valorise les valeurs européennes de base :

[...] languages are not a mere means of communication. They contribute to a better knowledge of other European cultures and have a real potential for a deeper understanding between European citizens. Multilingualism policy aims at ensuring multiculturalism, tolerance and European citizenship. Widespread general competence in foreign languages also plays its part in keeping xenophobia and intolerance at bay (Figel, 2005:3).

- Identitaires - le multilinguisme constitue la base d'une identité culturelle européenne : Each language deserves to be seen and treated as a language of culture - on account of its semantic and grammatical peculiarities as well as literary and other works written in this language. Looked at from this point of view, multilingualism is a value and as such a characteristic of European cultural identity (Commission of the European Communities, 2007: 17).

11 La construction identitaire de chaque citoyen, en tant qu'Européen, passe par le sentiment d'appartenance à une société plus large qui respecte les particularités de chacun :

Négliger une langue, c'est prendre le risque d'une désaffection de ses locuteurs à l'égard de l'idée européenne. Nul ne peut adhérer de tout cœur à l'ensemble 
européen s'il n'a le sentiment que sa culture spécifique, et d'abord sa langue, y est pleinement respectée, et que l'intégration de son pays à l'Union européenne contribue à épanouir sa langue propre et sa culture propre plutôt qu'à les marginaliser (Maalouf, $2008: 13$ ).

- Sociaux - le multilinguisme contribue au bien-être social et individuel («The various dimensions of multilingualism, above all the learning of languages, have the greatest significance for the good of society and for the well-being of individuals " (Commission of the European Communities, 2007: 22)), et atténue des attitudes de xénophobie et d'intolérance:

Our societies are becoming multicultural. But sometimes diversity is seen as a threat and communities live in isolation, triggering xenophobia. We must evolve into an intercultural society, where citizens of different backgrounds share a dialogue. And for dialogue, we need languages ! (Orban : $2008: 3$ ).

- Économiques - le multilinguisme favorise la Stratégie de Lisbonne et permet la construction d'une société plus compétitive, facilitant les échanges commerciaux, soutenant l'Europe en temps de crise et ouvrant de nouvelles chances (cf. Orban, 2007 et 2009).

Tous ces arguments sont résumés dans la communication de la Commission au Parlement européen $(2008: 3)$ :

The harmonious co-existence of many languages in Europe is a powerful symbol of the European Union's aspiration to be united in diversity, one of the cornerstones of the European project. Languages define personal identities, but are also part of a shared inheritance. They can serve as a bridge to other people and open access to other countries and cultures, promoting mutual understanding. A successful multilingualism policy can strengthen life chances of citizens: it may increase their employability, facilitate access to services and rights and contribute to solidarity through enhanced intercultural dialogue and social cohesion. Approached in this spirit, linguistic diversity can become a precious asset, increasingly so in today's globalised world.

13 Cette option pour la défense du multilinguisme a ainsi conduit à des mesures spécifiques pour le développement de la politique linguistique. En 2003, fut lancé un premier plan d'action pour 2004-2006:

[...] in 2003 the Commission launched the 2004-2006 action plan for the promotion of language learning and linguistic diversity. The aim of this plan was to promote multilingualism in Europe, in cooperation with the Member States and by means of specific measures in our education and training programmes (Orban, 2007: 7).

14 L'importance croissante de cette problématique, combinée au besoin d'élargissement des portefeuilles de la Commission européenne en raison de l'entrée de deux nouveaux États membres, a conduit, en 2007, à la nomination d'un commissaire chargé du multilinguisme :

It was the decision of President José Manuel Barroso to make "Multilingualism" part of the portfolio of one of the Commission members and to assign him responsibility for multilingualism in education, culture, interpretation, translation, and publications that set the signal for the development of a coherent and comprehensive EU language policy. The drive towards the development of such a policy gained considerable momentum when, on 1 January 2007, "Multilingualism" was made a separate portfolio, assigned to one of the new Commissioners, $\mathrm{Mr}$ Leonard Orban. (Commission of the European Communities, $2007: 5-6$ ).

15 Cependant, à partir de 2010, ce portefeuille a été supprimé, et le multilinguisme est une partie des attributions du Commissaire pour l'éducation, la culture, du multilinguisme et de la jeunesse. À l'heure actuelle, et depuis 2014, la nouvelle Commission présidée par Jean-Claude Junker a encore supprimé la mention "multilinguisme" du portefeuille 
spécifique de l'éducation, de la culture, des sports et de la jeunesse, dont le commissaire Tibor Navracsics est chargé. Le multilinguisme disparaît ainsi des priorités de ce commissaire, bien que la Direction générale de l'éducation et de la culture (DG EAC) soit encore explicitement responsable des politiques relatives à l'éducation, la culture, la jeunesse, les langues et les sports. Dans le cadre du programme Erasmus +, de cette Direction générale, l'apprentissage des langues et la diversité linguistique est encore l'un des objectifs spécifiques, et l'on affirme même :

Multilingualism is one of the cornerstones of the European project and a powerful symbol of the EU's aspiration to be united in diversity. Foreign languages have a prominent role among the skills that will help equip people better for the labour market and make the most of available opportunities (European Commission, 2015: 10).

Simultanément, le Conseil de l'Europe, une organisation intergouvernementale créée en 1949 et rassemblant aujourd'hui 47 Etats, dont les 28 membres de l'UE, a également entrepris un travail décisif dans la promotion du multilinguisme, en soutenant, concrètement, les politiques linguistiques européennes, notamment à travers l'action du Centre européen pour les langues vivantes. Son programme récent pour 2016-2019, "Languages at the heart of learning", réaffirme l'importance cruciale de la diversité linguistique comme support de la citoyenneté et ouvre de nouvelles possibilités pour le développement de "new solutions to the challenges in language education identified by our member states, through projects and think tanks » (ECML, 2016).

Toutefois, l'option politique en faveur du multilinguisme n'est sûrement pas pacifique et elle a été souvent remise en question au cours des dernières années, en particulier à partir de l'élargissement progressif de l'Union européenne. Le débat qui oppose les partisans du multilinguisme à ceux qui préconisent la construction d'un espace commun de communication basé sur l'utilisation de l'anglais comme lingua franca n'est pas clos. L'affirmation de Klippel (2001) - «There is universal agreement that Europe needs more than English» - ne correspond pas totalement à la réalité d'aujourd'hui. Il est vrai, comme l'affirme van Splunder (2014: 221), que « [t]he increasing use of English is clearly informed by neoliberal discourses on globalization, the emergence of a knowledge economy (in which language plays a crucial role) and the commercialization of higher education ». La naturalisation discursive sur le rôle unique de l'anglais comme langue de communication dans le monde est accompagnée par des discours académiques qui soutiennent ce point de vue, bien que la recherche récente dans ce domaine ${ }^{6}$ dévoile une réalité fort différente. De même, très récemment, Tremblay (2015 : 48) rappelle que

[...] ce qui caractérise le monde d'aujourd'hui c'est moins la tendance au monolinguisme que les progrès manifestes du plurilinguisme.

Il est important de souligner que la situation des langues sur Internet confirme ce constat. Ces progrès coexistent, certes, avec la suprématie de l'anglais. Mais l'anglais est passé dans le total des pages sur Internet de plus de $80 \%$ il y a dix ans à moins de $40 \%$ aujourd'hui. L'anglais n'est pas ou n'est plus en situation de monopole et si hégémonie il y a, celle-ci se trouve largement contestée dans les faits, alors même que l'on admet que la connaissance de l'anglais est une nécessité incontournable pour l'emploi à partir d'un certain niveau. Cette observation n'est pas contradictoire avec le constat précédent car, il est facile de comprendre qu'apprendre une langue ou plusieurs langues n'implique absolument pas un renoncement à sa propre langue. En tout cas, si cela peut arriver dans certains contextes, cela peut s'expliquer par d'autres raisons. Notons quand même que le plurilinguisme n'exclut pas l'anglais. 


\section{Les approches plurielles en Didactique des Langues Etrangères}

Intégrées dans le développement des politiques linguistiques européennes (et, plus particulièrement, des politiques linguistiques éducatives), et découlant très souvent de Projets financés dans le cadre des programmes Lingua (jusqu'en 2000), Socrates - Actions Lingua 1 et 2 (2000 - 2006) et Transversal LLP, Action clé 2 (2007 - 2013), les « approches plurielles» envisagent les langues en tant que manifestations diverses d'une entité unique, mais plurielle - la langue :

[p]ar définition, on appellera approche plurielle toute approche mettant en œuvre des activités impliquant à la fois plusieurs variétés linguistiques et culturelles. En tant que telle, une approche plurielle se distingue d'une approche singulière, dans laquelle le seul objet d'attention est une langue ou une culture particulière, prise isolément (Candelier, 2008: 68).

Cette dénomination couvre un petit ensemble méthodologique composé aujourd'hui par trois propositions didactiques dont les finalités spécifiques sont différentes :

- Intercompréhension ;

- Eveil aux Langues (Candelier, 2008 ; De Pietro, 2008 ; Candelier \& De Pietro, 2008);

- CLIL/EMILE (Marsh, 2001) ou enseignement bilingue (Gajo, 2008).

\subsection{Intercompréhension}

Historiquement, le terme intercompréhension (IC) surgit pour décrire une forme de communication entre les locuteurs de variétés dialectales de la même langue. En fait, la première occurrence du terme se doit à Jules Ronjat, 1913, dans le cadre de son étude Essai de syntaxe des parlers provençaux modernes. En se penchant sur la distinction entre les langues et dialectes, Ronjat a conclu que la présence ou l'absence d'intercompréhension dans des situations de communication concrètes conduirait à affirmer que les sujets parlaient, respectivement, des variantes de la même langue ou des langues différentes (cf. Escudé, 2010 ; Ollivier, 2010, 2013, sous presse). Ainsi l'IC se place-t-elle initialement sur le plan de l'observation empirique (Palmerini \& Faone, 2010), servant à designer des phénomènes de compréhension réciproque spontanée entre des sujets parlants ou des groupes de sujets parlants (Ollivier, 2010).

Plus tard, pendant le $\mathrm{xx}^{\mathrm{e}}$ siècle, cette désignation s'est élargie à des phénomènes similaires dans le contexte des contacts interlinguistiques. En fait, l'IC est rapportée dans une tradition séculaire de pratiques de communication entre les habitants des régions frontalières ou insérée dans les expériences des voyageurs de tous les temps; le phénomène correspond à des processus naturels et spontanés mis en œuvre par des individus 'communs' dans les situations de contacts exolingues. Ainsi certaines personnes sont-elles capables d'effectuer spontanément des processus d'IC lorsqu'elles sont confrontées à une langue étrangère inconnue ou peu connue. Il s'agit, donc, d'« un vécu banal et quotidien pour des acteurs bien réels, attesté au long de l'histoire » (Grin, 2008: 18). Et comme Bernard Cassen, beaucoup de ceux qui vivent au quotidien dans des environnements plurilingues pourront affirmer : «J'ai sans le savoir, pratiqué de manière précoce l'intercompréhension et la création terminologique sauvage » (Cassen, 2008: 13). Comme le conclut Tyvaert (2008: 274), «[1'] intercompréhension se présente finalement 
comme une méthode naturelle, qui met en forme des habiletés sémiotiques latentes que sait mobiliser, plus ou moins bien, tout sujet parlant quand il migre d'un espace linguistique à un autre ».

Du point de vue de sa légitimité dans le domaine de la Didactique des Langues, l'IC se situe clairement au sein des propositions du CECR. S'il est vrai que, comme le rapportent Escudé \& Janin (2010 : 16), l'IC n'est pas explicitement mentionnée dans le texte du CECR, elle s'intègre totalement dans la perspective de l'apprentissage pluriel prévu dans cette œuvre de référence. En effet, l'IC est inscrite dans la conception de plurilinguisme présentée par le CECR (2001:11) :

Dans des situations différentes, un locuteur peut faire appel avec souplesse aux différentes parties de cette compétence pour entrer efficacement en communication avec un interlocuteur donné. Des partenaires peuvent, par exemple, passer d'une langue ou d'un dialecte à l'autre, chacun exploitant la capacité de l'un et de l'autre pour s'exprimer dans une langue et comprendre $\underline{\text { l'autre }}^{7}$. D'aucun peut faire appel à sa connaissance de différentes langues pour comprendre un texte écrit, voire oral, dans une langue a priori «inconnue ", en reconnaissant des mots déguisés mais appartenant à un stock international commun.

D'autre part, elle s'inscrit également dans la possibilité de prévoir, dans le cadre de l'apprentissage plurilingue, la reconnaissance - même à un niveau institutionnel - de compétences partielles :

La reconnaissance « officielle » de compétences partielles peut aller dans ce sens [... ] On aurait toutefois aussi intérêt à prendre en compte et à valider en tant que telle une compétence plurielle à même de jouer de plusieurs langues ou de plusieurs cultures. Traduire (ou résumer) d'une deuxième langue étrangère vers une première langue étrangère, participer à un échange oral plurilingue ${ }^{8}$, interpréter un phénomène culturel en relation à une autre culture sont des activités d'interaction ou de médiation (au sens donné ici à cette notion) qui ont leur place dans des pratiques effectives. À bien des égards, c'est aussi le profil plurilingue et pluriculturel et les capacités de gestion d'un tel répertoire que les certifications devraient également permettre d'apprécier et de valoriser (CECR, 2001 : 133).

D’une façon encore plus explicite: "La compétence partielle dans une langue donnée peut concerner des activités langagières de réception (mettre l'accent par exemple sur le développement d'une capacité de compréhension orale ou écrite)» (idem: 106). Ou encore (ibidem) :

Rien n'interdit de penser que ces visées particulières portant, à un moment donné, sur un secteur ou un type particulier de compétence, une mise en place ou un développement de compétence partielle, permettent l'installation ou le renforcement d'une compétence plurilingue et pluriculturelle transversale. En d'autres termes encore, la poursuite d'un objectif partiel peut trouver place dans un projet d'ensemble pour l'apprentissage.

Le développement de projets et de matériaux qui a systématiquement marqué ce domaine depuis les années 1990, notamment en ce qui concerne les langues romanes, devrait servir à la dissémination de cette approche et à son inclusion dans l'espace formel de l'apprentissage scolaire. Toutefois, l'immobilisme institutionnel qui caractérise les systèmes éducatifs représente encore aujourd'hui un obstacle à franchir. 


\section{2. Éveil aux Langues}

26 L'approche Éveil aux Langues, développée initialement dans le cadre du projet Evlang (Candelier, 2003), s'adresse à un public de jeunes enfants et a pour objectif le développement

- des aptitudes d'observation et de raisonnement métalinguistique ;

- des savoir-faire utiles pour l'apprentissage des langues ;

- des attitudes d'ouverture à la diversité linguistique et culturelle ;

- des savoirs relatifs aux langues et à leur diversité (Candelier \& De Pietro, 2008 : 149).

Sur ces objectifs, De Pietro (2008: 205) ajoute encore :

Plus généralement, les démarches d'éveil aux langues visent à développer chez les apprenants des attitudes plus ouvertes envers les langues - en particulier celles qu'ils doivent apprendre à l'école et celles qui sont présentes dans la salle de classe - des aptitudes d'écoute, d'observation, d'analyse qui leur sont utiles dans leurs différents apprentissages linguistiques, et des connaissances à propos du langage et des langues qui ont entre autres pour fonction de fonder, d'aider à comprendre, les aptitudes et attitudes à développer. Autrement dit, il ne s'agit pas en premier lieu d'« enseigner » les langues prises en compte mais bien de construire, au moyen ou grâce à la diversité des langues, différentes ressources et compétences pertinentes pour « fonctionner» dans un environnement plurilingue.

Cette longue citation contient les éléments principaux qui caractérisent l'Éveil aux Langues et qui le distinguent de l'IC:

- il s'agit d'approche propédeutique à un apprentissage plus complet de la langue ;

- il vise à développer prioritairement des attitudes et des compétences nécessaires à des apprentissages postérieurs ;

- il est principalement destiné à des enfants en début de scolarisation (cf. De Carlo, 2015 : 99).

L'Éveil aux Langues pourrait ainsi garantir, dès le plus jeune âge, l'ouverture à la diversité linguistique et culturelle, essentielle au développement d'attitudes menant à la citoyenneté européenne. Nous regrettons que cette approche n'ait pas été prise en compte lors de la reformulation des programmes d'anglais au niveau du $1^{\text {er }}$ cycle, au Portugal.

\subsection{EMILE (Enseignement d'une Matière par l'Intégration d'une Langue Etrangère)}

30 Il s'agit d'une approche systématisée dans le domaine de l'enseignement bilingue. Consistant, comme son nom l'indique, à l'apprentissage simultané d'une langue étrangère et de contenus scolaires non linguistiques, son intérêt

réside exactement dans le fait que les deux apprentissages procèdent simultanément et que les matières sont étudiées avec et à travers une autre langue plutôt que simplement dans une autre langue. La distinction est de taille et jette une lumière sur les rapports entre verbalisation et acquisition des savoirs (idem : 101).

On pourra ainsi conclure que cette approche présente des avantages significatifs dans un cadre général d'apprentissage : « The experience available shows that both linguistic and content subject competence can be promoted within this integrated concept more effectively than when content and language are taught in isolation" (Wolff, 2001: 47). L'approche EMILE s'insère donc dans une didactique multi-intégrée de L1, L2 et de 
disciplines non-linguistiques (cf. Gajo, 2008: 138) développée au sein des institutions scolaires.

Les expériences d'EMILE au Portugal sont encore insuffisantes, même si depuis 2003 les sections européennes - notamment celles de français - connaissent un véritable essor.

\section{Un espoir pour l'avenir}

Les approches plurielles constituent une base possible pour que le plurilinguisme des citoyens - plus particulièrement des citoyens portugais - puisse sortir de la sphère de l'utopie pour devenir une réalité. Sa reconnaissance institutionnelle serait un gage d'engagement sérieux visant un autre regard vers la diversité linguistique et culturelle, un autre vécu de l'Europe et le refus l'imposition d'une langue unique.

Par rapport à la langue française, elles pourraient apporter un renouvellement méthodologique, un nouvel essor des publics 'égarés', un marketing efficace en Europe et ailleurs (dont la DGLFLF a si bien su profiter en embrassant la cause de l'Intercompréhension) et une identité spécifique au sein d'une famille linguistique et culturelle. Libérée de son passé (et des préjugés qui longtemps en ont découlé), la langue française retrouverait ainsi, en Europe, son espace de médiation humaniste, de culture, de poésie, de savoir, mais aussi de modernité, de recherché scientifique, de communication et de rencontre(s).

Du point de vue de sa politique linguistique, la France prend actuellement conscience des mesures à prendre. Outre l'action efficace permanente da la DGLFLF, l'Agence Nationale Erasmus+ semble avoir reconnu le besoin de travailler pour le plurilinguisme : en 2015 et 2016, elle a financé la mise en œuvre de deux grands Partenariats Stratégiques concernant des approches plurilingues de l'apprentissage, contrariant l'indifférence générale portée par d'autres pays aux projets linguistiques.

Mais il appartient aussi aux acteurs du terrain - enseignants de français à tous les niveaux - de garantir l'avenir des langues et de la diversité culturelle et... l'avenir du français.

\section{BIBLIOGRAPHIE}

BELLINI, Paolo (2008). «Identité Européenne, Pouvoir Politique et Globalisation ». In Revue internationale de philosophie en ligne, mai 2008 an III n 5 [disponible le 10.10.2016]. <URL : http:// www.metabasis.it/articoli/5/5_Bellini.pdf>.

BERTHOUD, Anne-Claude \& BURGER, Marcel (orgs.) (2014). Repenser le rôle des pratiques langagières dans la constitution des espaces sociaux contemporains. Bruxelles : De Boeck.

BITTENCOURT, Bernardete (2008). « Dinâmicas sociais contemporâneas : redes, capital social e desenvolvimento sustentável ». In e-cadernos ces, nำ 2/2008, Novos Mapas para as Ciência Sociais e Humanas - artigos pré colóquio. [disponible le 10.10.2016]. <URL : https://eces.revues.org/1418>. 
CANDELIER, Michel (2008). «Approches plurielles, didactiques du plurilinguisme : le même et l'autre ». Recherches en didactique des langues - L'Alsace au coeur du plurilinguisme. Les Cahiers de l'Acedle, vol. 5, n 1, 65-90.

CANDELIER, Michel (org.) (2003). Evlang - l'éveil aux langues à l'école primaire - Bilan d'une innovation européenne. Bruxelles : De Boek - Duculot.

CANDELIER, Michel \& et DE PIETRO, Jean-François. (2008). « Éveil aux langues et argumentations curriculaires : choix européens et fondements empiriques ». In AUDIGIER F. \& TUTIAUX-GUILLON N. (orgs.). Compétences et contenus - Les curriculums en question. Bruxelles : De Boeck Université, pp. 147-162.

CAPUCHO, Maria Filomena (2006). «Sobre Línguas e Culturas », in Rev. Veredas online, número especial Portugal, vol. 18-19 Universidade Federal de Juiz de Fora, Brasil. [disponible le 10.10.2016]. <URL : http://www.ufjf.br/revistaveredas/files/2009/12/artigo094.pdf>

CASSEN, Bernard (2008). « Préface ». In V. CONTI \& F. GRIN (orgs.). S'entendre entre langues voisines : vers l'intercompréhension. Chêne-Bourg: Georg Editeur, pp. 13-15.

COMMISSION OF THE EUROPEAN COMMUNITIES (2007) Final Report - high level group on multilingualism. [disponible le 4.04.2016]. <URL : http://bookshop.europa.eu/en/high-level-group-onmultilingualism-pbNC7807451/>

COMMISSION OF THE EUROPEAN COMMUNITIES (2008). An Inventory of Community Actions in the Field of Multilingualism and Results of the Online Public Consultation. [disponible le 10.10 2016]. <URLhttp:// register.consilium.europa.eu/doc/srv?l=EN\&f=ST\%2013253\%202008\%20ADD\%201>

CONSEIL DE L'EUROPE (2001). Cadre européen commun de référence pour les langues vivantes : apprendre, enseigner, évaluer. Paris : Editions Didier.

DE CARLO, Maddalena (2015). Le développement de compétences métalinguistiques par les approches plurielles. In D. SPIȚĂ, M. LUPU, D. NICA \& I. NICA (orgs.). Les approches plurielles dans l'éducation aux langues : l'intercompréhension, en présence et en ligne. Iasi : Editura UniversităȚii Alexandru Ioan Cuza, pp. 93-106.

DE PIETRO, Jean-François. (2008). « De l'éveil aux langues à l'intercompréhension et vice-versa ». In V CONTI, \& F. GRIN (orgs.). S'entendre entre langues voisines : vers l'intercompréhension. Chêne-Bourg : Georg Editeur, pp. 197-226.

ESCUDE, Pierre (2010). « Origine et contexte d'apparition du terme intercompréhension dans sa première attestation (1913) chez le linguiste Jules Ronjat (1864 - 1925)». In C. FERRÃO TAVARES et Ch. OLLIVIER (orgs.). o conceito de Intercompreensão : origem, evolução e definições. RedinterIntercompreensão, 1, 103-124.

ESCUDE, Pierre \& JANIN, Pierre (2010). Le point sur l'intercompréhension, clé du plurilinguisme. Paris: Clé International.

European centre for modern languages of the council of Europe (2016). The European Languages Gazette Newsletter, Nr. 30 - February/March 2016.

EUROPEAN COMMISSION (2015). Erasmus+ Programme Guide 2016. [disponible le 10.10.2016]. <URL : http://ec.europa.eu/programmes/erasmus-plus/sites/erasmusplus/files/files/resources/ erasmus-plus-programme-guide_en.pdf_

FIGEL, Jan (2005) « Preface ». In Eurydice European Unit. Key data on teaching languages at school in Europe. [disponible le 10.19 2016]. <URL http://www.indire.it/lucabas/lkmw_file/eurydice/// Key_data_languages_EN.pdf> 
FRIEDMAN, Thomas (2000). The Lexus and the Olive Tree - Understanding Globalization. New York : First Anchor Books Ed.

GAJO, Laurent (2008). «L'intercompréhension entre didactique intégrée et enseignement bilingue ». In V. CONTI \& F. GRIN (orgs.). S'entendre entre langues voisines : vers l'intercompréhension. Chêne-Bourg : Georg Editeur, pp. 131-150

GRIN, François (2008). « Pourquoi l'intercompréhension? ». In V. CONTI \& F. GRIN (orgs.). S'entendre entre langues voisines : vers l'intercompréhension. Chêne-Bourg : Georg Editeur, pp. 17 - 30.

KLIPPEL, Frederika (2001.) Innovation and tradition - foreign language teaching in Europe at the beginning of the new millenium, conference tenue lors de "Languages: Promoting Good Practice ", Rotterdam, November 2001 (mimeo).

MAALOUf, Amin (org.) (2008). Un Défi Salutaire - Comment La Multiplicité Des Langues Pourrait Consolider l'Europe - Propositions du Groupe des Intellectuels pour le Dialogue Interculturel constitué à l'initiative de la Commission Européenne. Bruxelles. [disponible le 10.10 2016]. <URL: http:// frankofonia.pl/download/images/dokumenty/report_fr.pdf> MEISSNER, Franz-Joseph (2007). « Didactique du plurilinguisme et développements scolaires ». In CAPUCHO F., A. ALVES, P. MARTINS, Ch. DEGACHE \& M. TOST (orgs.) (2007). Diálogos em Intercompreensão ( $2^{a}$ edição - CdRom). Lisboa : Universidade Católica, pp. 195 - 216.

MUNSHI, Surendra (2006). « Será o mundo plano ? ». In Pacheco, C. et al. o Estado do Mundo. Lisboa : Ed. Temas e Debates. Fundação Calouste Gulbenkian, pp. 257 - 285.

OLLIVIER, Christian (2010). « Représentations de l'intercompréhension chez les spécialistes du camp ». In C. FERRÃO TAVARES \& Ch. OLLIVIER (orgs.). O conceito de Intercompreensão : origem, evolução e definições. Redinter-Intercompreensão, 1, 47-70.

OLLIVIER, Christian (2013). « Tensions Épistémologiques en Intercompréhension ». Recherches en Didactique des Langues et des Cultures : Les Cahiers de l'Acedle 10, 1, 5-27.

OLLIVIER, Christian (sous presse). «L'interproduction : entre foreigner talk et spécificité en intercompréhension ». In Ch. DEGACHE \& S. GABARINO (orgs.). Itinéraires pédagogiques de l'alternance des langues : l'intercompréhension. Grenoble : ELLUG, pp. 233-244.

ORBAN, Leonard (2007). European multilingualism policy - discours tenu lors de l'Europe Brussels International le 17 décembre 2007 [disponible le 10.10 2016]. <URL : http://ec.europa.eu/archives/ commission_2004-2009/orban/news/docs/ speeches/071217_European_multilingualism_policy/071217_European_multilingualism_policy_en.pdf $>$

ORBAN, Leonard (2008). Why and where do languages matter? Towards a comprehensive strategy for multilingualism, Ministerial Conference on "Promoting Multilingualism: a shared commitment", Brussels, 15 February 2008 [disponible le 10.10 2016]. <URL: http://europa.eu/rapid/ pressReleasesAction.do? reference=SPEECH/08/83\&format=PDF\&aged=1\&language=EN\&guiLanguage> ORBAN, Leonard (2009). Multilingualism in the EU: promoting intercultural dialogue, linguistic diversity and language, ALTE Conference, Santiago de Compostela, the 24th of April 2009 [disponible le 10.10 2016]. <URL: http://ec.europa.eu/archives/commission_2004-2009/orban/news/docs/ speeches/090424_ALTE_conference/ALTE_conferencere_EN.pdf> 
PALMERINI, Monica \& FAONE, Serena (2010). «Sul cammino verso l'intercomprensione. Una riflessione epistemologica ». In C. FERRÃo TAVARES \& Ch. OLLIVIER (orgs.). 0 conceito de Intercompreensão : origem, evolução e definições. Redinter-Intercompreensão, 1, 187-222.

TYVAERT, Jean-Emmanuel (2008). « Pour une refondation de la didactique des langues sur la base de l'intercompréhension ». In V. CONTI \& F. GRIN (orgs.). S'entendre entre langues voisines : vers l'intercompréhension. Chêne-Bourg : Georg Editeur, pp. 251-276.

TREMBLAY, Christian. (2015). « Le plurilinguisme européen sur le fil du rasoir ». In D. SpiȚă, M. Lupu, D. Nica \& I. Nica (orgs). Les approches plurielles dans l'éducation aux langues:

l'intercompréhension, en présence et en ligne. Iasi : Editura UniversităȚii Alexandru Ioan Cuza, pp. 43 $-68$.

UNGER, Johann.W, KRZYZANOWSKI Michał \& WODAK, Ruth (2014). Multilingual encounters in Europe's institutional spaces. London: Bloomsbury.

VAN SPLUNDER, Frank (2014). « Negotiating multilingualism in Flemish higher education ». In J.W. UNGER, M. Krzyżanowski. \& R. Wodak, Multilingual encounters in Europe's institutional spaces. London: Bloomsbury, pp. 221-242.

VERTOVEC, Stephen (2006). The Emergence of Super-diversity in Britain. Working Paper No. 25. Oxford: Centre for Migration, Policy and Society, University of Oxford.

WOLFF, Dieter (2001). On the importance of CLIL in the context of the debate on plurilingual education in the European Union. In MARSH, D. (org.) CLIL/EMILE The European dimension-Actions, Trends and Foresight Potential. University of Jyväskylä, Finland: UniCOM, Continuing Education Centre, pp. $47-48$.

\section{NOTES}

1. Parmi lesquels plus d'un million de réfugiés, un chiffre en croissance continue depuis 2015.

2. Si l'on compte encore le Royaume Uni, dont Brexit n'est pas encore conclu.

3. Valuing All Languages in Europe. European Centre for Modern Languages. 2nd Medium-term programme 2004-2007 (http://www.ecml.at/mtp2/VALEUR/html/ Valeur_E_news.htm\#March2007).

4. Texte de la Déclaration de la Présidence du Conseil européen disponible sur <http:// www.eng.unibo.it/NR/rdonlyres/F3A0A962-3AB9-478B-8FCF-398A4F530448/25824/

BarcelonaEuropeanCouncilConclusion.pdf>

5. Je distingue clairement les termes multilinguisme (concernant la politique linguistique de base de la Commission Europénne, visant le respect de la pluralité linguistique dans l'Union et dans ses Etats membres) et plurilinguisme (compétences linguistiques plurielles des citoyens) : « La promotion du pluriguisme est un but central de la politique européenne des langues, but légitimé par le multilinguisme des états membres » (Meissner, 2007 : 196). Cf. également Capucho, 2006.

6. Je me rapporte très spécifiquement aux résultats du projet Dylan (Language Dynamics and Management of Diversity), développé entre 2006 et 2011 e à une vaste production académique qui en découle (cf. UNGER, KRZYŻANOWSKI \& WODAK, 2014, BERTHOUD \& BURGER, 2014, entre autres).

7. C'est moi qui le souligne.

8. Idem. 


\section{RÉSUMÉS}

Les politiques linguistiques définies par le Conseil Européen et la Commission Européenne depuis la Déclaration de Lisbonne, en 2000, visent ouvertement le développement du plurilinguisme des citoyens et le multilinguisme des états. Ces politiques souhaitent contrecarrer l'indéniable imposition économique et sociale de l'anglais, vu comme lingua franca pour toute communication internationale, menaçant la diversité linguistique et culturelle de l'UE. Dans cet article, j'esquisserai brièvement l'évolution de ces politiques et j'analyserai ensuite leurs apports au développement du français dans l'espace européen. Je m'arrêterai plus longuement sur l'analyse $\mathrm{du}$ rôle que les approches plurielles peuvent jouer par rapport à l'intégration du français dans sa famille linguistique - les langues romanes - dont l'apprentissage pourra revitaliser l'intérêt social porté à la langue française et lui redonner un nouvel élan.

Language policies defined by the European Council and the European Commission since the Lisbon Declaration, in 2000, openly target the development of the plurilinguism of citizens and the multilingualism of states. These policies aim at thwarting the undeniable economic and social imposition of English, seen as a lingua franca for all international communication and threatening the linguistic and cultural diversity of the EU. In this paper, I will outline briefly the evolution of these policies, and I will then analyze their contributions to the development of French in the European area. I will emphasize the role that plural approaches can play for the integration of the French language in its family - Romance languages - allowing to revitalize the social interest in the French language and give it a new impulse.

\section{INDEX}

Keywords : language policies, multilingualism, plurilingualism, French, plural approaches Mots-clés : politiques linguistiques, multilinguisme, plurilinguisme, français, approches plurielles

\section{AUTEUR}

\section{FILOMENA CAPUCHO}

Universidade Católica Portuguesa - CECC

fcapucho[at]gmail.com 